Original paper

\title{
Exploring TOF capabilities of PET detector blocks based on large monolithic crystals and analog SiPMs
}

\author{
Efthymios Lamprou*, Antonio J. Gonzalez, Filomeno Sanchez, Jose M. Benlloch \\ Instituto de Instrumentación para Imagen Molecular (I3M), Centro Mixto CSIC — Universitat Politècnica de València, 46022 Valencia, Spain
}

\section{A R T I C L E I N F O}

\section{Keywords:}

TOF-PET

Monolithic crystal

ASIC

SiPMs

\begin{abstract}
A B S T R A C T
Monolithic scintillators are more frequently used in PET instrumentation due to their advantages in terms of accurate position estimation of the impinging gamma rays both planar and depth of interaction, their increased efficiency, and expected timing capabilities. Such timing performance has been studied when those blocks are coupled to digital photosensors showing an excellent timing resolution.

In this work we study the timing behaviour of detectors composed by monolithic crystals and analog SiPMs read out by an ASIC. The scintillation light spreads across the crystal towards the photosensors, resulting in a high number of SiPMs and ASIC channels fired. This has been studied in relation with the Coincidence Timing Resolution (CTR). We have used LYSO monolithic blocks with dimensions of $50 \times 50 \times 15 \mathrm{~mm}^{3}$ coupled to SiPM arrays $\left(8 \times 8\right.$ elements with $6 \times 6 \mathrm{~mm}^{2}$ area) which compose detectors suitable for clinical applications.

While a CTR as good as 186 ps FWHM was achieved for a pair of $3 \times 3 \times 5 \mathrm{~mm}^{3}$ LYSO crystals, when using the monolithic block and the SiPM arrays, a raw CTR over 1 ns was observed. An optimal timestamp assignment was studied as well as compensation methods for the time-skew and time-walk errors. This work describes all steps followed to improve the CTR. Eventually, an average detector time resolution of 497 ps FWHM was measured for the whole thick monolithic block. This improves to 380 ps FWHM for a central volume of interest near the photosensors. The timing dependency with the photon depth of interaction and planar position are also included.
\end{abstract}

\section{Introduction}

Since the development of the first Positron Emission Tomography (PET) scanners already back in 80's, several efforts have been devoted to provide an accurate timing determination of the $511 \mathrm{keV}$ annihilation photons [1-3]. This information, typically known as Time-OfFlight (TOF), is directly impacting improvements in the contrast of the reconstructed medical images [4]. Unarguably, the continuous research in this field and the potential achievement in the so-called Coincidence Time Resolution (CTR) to values below 100 ps Full-Width-at-HalfMaximum (FWHM), will lead to a certain technological revolution of both clinical and pre-clinical PET practice [5].

In order to achieve an excellent time resolution in PET instrumentation, several factors need to be considered, such as an efficient photosensor exhibiting a fast rise time, high quantum efficiency (QE) and relatively high gain [6]. A photosensor with these characteristics and very often used in gamma ray detectors is the photomultiplier tube (PMT). They have been used in several applications, demonstrating also their feasibility to be essential components in TOF-
PET detectors [6,7]. An alternative photosensor device is the silicon photomultiplier (SiPM) $[8,9]$. Latest works show that SiPMs are gaining ground over the PMTs in gamma-ray detectors due to their compact size, their compatibility to magnetic fields, and a high photodetection efficiency (PDE). Shortly, the operating principle of SiPMs is based on the sum of all internal single-photon avalanche diodes (SPADs). This inherently introduces some uncertainty in the event timestamp generation. An alternative approach to SiPM was introduced by Philips Digital Photon Counting (Aachen, Germany), with the so-called digital silicon photomultipliers (dSiPMs). In their architecture, each cell is composed by its independent SPAD and its readout electronics, and it is capable of detecting exactly one photon. Detailed descriptions of their working principle and characteristics can be found $[10,11]$.

Another key element in the performance of a detector block is the scintillation crystal. Recent advances in this area have well enabled the development of TOF-PET systems [12-14]. A scintillator crystal suitable for TOF-PET detectors, besides high stopping power, must also exhibit high initial photon intensity [4]. This characteristic can be achieved by an adequate light output and a short decay time.

\footnotetext{
* Corresponding author.

E-mail address: e.lamprou@i3m.upv.es (E. Lamprou).
} 

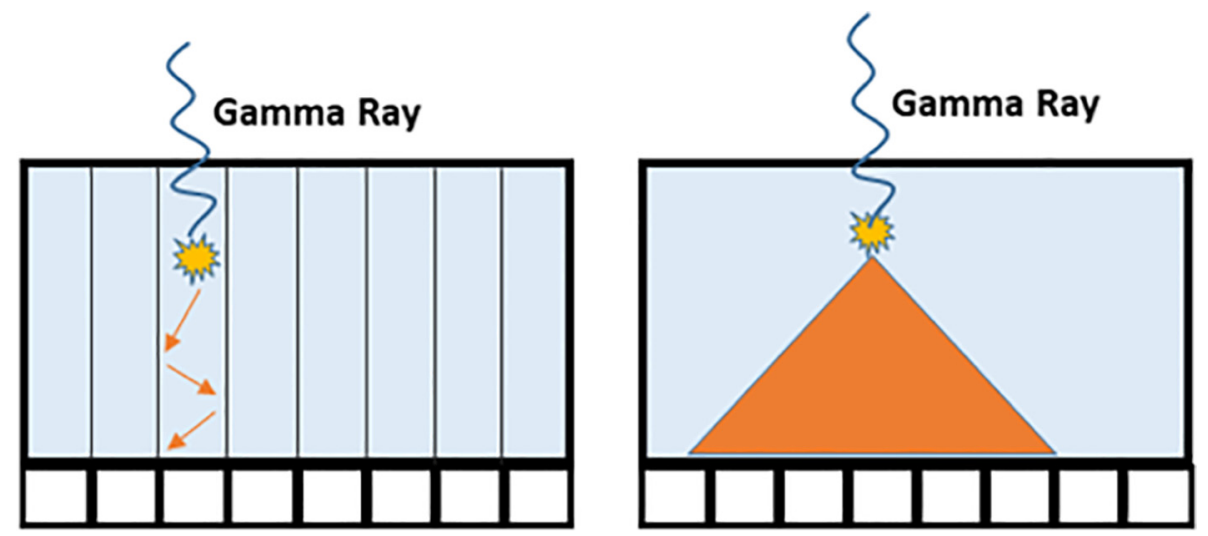

Fig. 1. Representation of the scintillation light distribution for one gamma event inside a pixelated crystal (left) and a monolithic block (right).

Nowadays several crystal types and compositions suitable for TOF are available [15]. There are mainly two types of scintillators used in gamma ray detectors namely pixelated crystals and monolithic blocks. Both types are briefly described below, while emphasis in the present work is given to the second type.

When aiming to achieve a very good timing resolution, the claimed most efficient approach is to use arrays of pixelated crystals with pixel dimensions that match that of the photosensor element active area, dubbed as one-to-one coupling. An example of this approach can be seen in Fig. 1 left. In this configuration, after some internal reflections of the generated optical photons inside the crystal pixel, they eventually exit and are collected mainly by a single photosensor element with no significant losses to neighbor photosensors. This allows one to collect high amounts of visible photons at a given short time frame. The main degradation observed in this case is some delay of the optical photons to reach the photosensors due to the light transfer efficiency (LTE) and the light transfer time spread (LTTS) $[6,16]$. Moreover, the detector spatial resolution is limited to the pixel size. The deterioration in the CTR increases for longer light paths meaning for thicker scintillators. However, this difference does not exceed some tenths of picoseconds. An alternative detector configuration towards improving the detector block spatial resolution makes use of crystal arrays with pixel sizes smaller than the photosensor elements, implying scintillation light sharing among few photosensors [17]. Optical lightguides are employed avoiding accumulation of events in a single photosensor. This approach tends to degrade the CTR due to the spread of the optical photons among neighbor photosensor elements.

Detector block configurations which make use of monolithic crystals provide some advantages when compared to pixelated crystals and, therefore, make them good candidates for PET applications [18-21]. The crystal thickness and geometry, as well as treatments on the walls vary depending on the application.

In monolithic blocks the scintillation photons are isotropically emitted travelling straight in all directions, differently from the pixelated crystal case in which the optical photons are trapped inside bouncing on the walls until they reach one photosensor. The light spread in the monolithic block permits an accurate position decoding of the gamma ray impact, being a convenient choice for a high intrinsic detector spatial resolution [22]. In addition to the position, a monolithic scintillator could ideally show a better timing performance compared to a pixelated one, due to the fact that the generated optical photons are not suffering from the aforementioned internal reflections inside the crystal pixel introducing time delays. However, the wide spread of the scintillation light does not facilitate the collection of a high number of photons at each single photosensor element in a very short time, which is mandatory for a good TOF. The poor collection of optical photons and the resulting low Signal-To-Noise ratio (SNR) for each channel, leads to noise and false signal triggering. In order to reach a good CTR, it is critical to use high performance readout electronics especially sensitive to the first photoelectrons. Ideal candidates for this purpose are the aforementioned dSiPMs, but also novel Application Specific Integrated Circuits (ASICs) specifically designed with low noise electronics.

Few works have been published showing that dSiPMs can successfully be combined with monolithic blocks to provide accurate TOF information even below 200 ps FWHM [23,17]. Due to their operating principle, these photosensors can be sensitive to the very first photoelectrons while keeping the noise level very low. Typically, this is achieved by operating them at low temperatures of $-20^{\circ} \mathrm{C}$, while at the same time they show the capability to disable microcells with higher levels of noise.

In this work, we explore the limits, in terms of timing resolution, when large and thick continuous crystals are read by analog SiPMs and ASICs. Emphasis has been given in analyzing the contribution of all photosensors which are involved in each generated scintillation distribution, aiming to get a better understanding of the light shape and its relevance with respect to the timing information. Evaluation results as well as methods to improve the CTR are being presented and discussed, aiming to shed light on the limits of timing resolution for this kind of detector configurations.

\section{Materials and methods}

\subsection{ASIC readout}

We have selected an ASIC to read, digitize and process all photosensors signals. All photosensors were individually read out avoiding reduction schemes introducing noise or additional delays in the timepaths of the signals. The ASIC used all throughout the measurements was the TOFPET2 (PETsys, Portugal). This particular chip can read up to 64 channels, and for each of them includes charge integration Analog-To-Digital Converters (ADCs), and Time-To-Digital Converters (TDCs) with $30 \mathrm{ps}$ binning. Inside the ASIC, the incoming signal is evaluated by two analog circuit schemes, before it becomes a valid gamma signal. The first one is related to the timing of the signal and is composed by two discriminators. The first discriminator, namely $v t h \_t 1$, uses a very low voltage threshold which typically corresponds to few photoelectrons and is designed to start the process. The output of this discriminator is fed into an AND gate after a programmable delay. To the same AND gate, the output of the second discriminator ( $\left.v t_{-}+2\right)$ is fed. $v$ th $t 2$ is set to a higher voltage threshold in order to discard dark counts without introducing any dead time to the system. The output of the AND gate results in a trigger signal which generates the timestamp using a $200 \mathrm{MHz}$ clock. The second circuit scheme is based on a discriminator ( $v$ th_e $e$ ) designed to discard pulses with relatively low amplitude and is operated as the energy threshold. Only when the three 
thresholds are met, a gamma-ray event is considered valid. Further information about the ASIC and the DAQ system can be found in references $[24,25]$, among others.

\subsection{SiPM photosensors}

Two types of SiPM photosensors were used. A pair of SiPMs with $3 \times 3 \mathrm{~mm}^{2}$ active area (PA3325 model, KETEK, Germany) configured at a bias voltage of $31 \mathrm{~V}$ were tested with small pixel crystals. Other experiments were carried out using two $8 \times 8 \mathrm{SiPM}$ arrays with $6 \times 6 \mathrm{~mm}^{2}$ active area each (ON-Semi, J-series model). The wide total active area of these arrays suggested them as good candidates for their integration in clinical TOF-PET systems and especially in combination with large monolithic crystals [26]. Those arrays have an active coverage area of $92 \%$ permitting the collection of high amounts of scintillation photons and, thus, improving the SNR. The larger capacitance of SiPMs with $6 \mathrm{~mm}$ will not significantly influence the CTR when combined with monolithic blocks, as the expected uncertainty due to the light spread might be larger [27]. Those SiPMs arrays were operated at two bias voltages, 29 and $30.5 \mathrm{~V}$, depending on the experiment.

\subsection{Detector set-ups}

Two types of experiments were designed. First, pixelated crystals following the one-to-one coupling were tested. The aim was to characterize the ASIC and the whole DAQ system performance. A coincidence measurement was carried out using the PA3325 SiPM sensors coupled to LYSO crystals wrapped with Teflon of $3 \times 3 \times 5 \mathrm{~mm}^{3}$. Thereafter, experiments were carried out with two photosensor elements from the J-Series arrays and two LYSO crystal pixels covered with Enhanced Specular Reflector (ESR) of $6 \times 6 \times 15 \mathrm{~mm}^{3}$. For both experiments, the ASIC discriminators were set to their default values, that means $v$ th $t 1=20, v$ th $t 2=20$ and $v$ th $e=15$, respectively.

The monolithic LYSO crystals had dimensions of $50 \times 50 \times 15 \mathrm{~mm}^{3}$, matching the SiPM array dimensions. These crystals were treated with black paint in the four lateral walls in order to avoid undesired internal reflections which typically influence the spatial resolution. A retroreflector layer was added to the entrance face
(Fig. 2 top-left). This particular optical element bounces back the light towards the emission point, improving the light collection at the photosensors while preserving the light distribution [20].

For the evaluation of the monolithic blocks we first studied one detector block against a reference detector composed by an individual LYSO pixel of $6 \times 6 \times 15 \mathrm{~mm}^{3}$ coupled to one photosensor element of an identical SiPM array (Fig. 2 bottom-right). This approach provides an optimal characterization of the performance of the monolithic crystal, as it exhibits the minimum uncertainty in terms of timing. The two detectors were independently configured in terms of SiPM bias and thresholds. For the reference pixel-based detector, we used the same configuration as for the initial one-to-one coupling experiments $(29 \mathrm{~V}$ and default thresholds). However, the detector with the monolithic block was set to $30.5 \mathrm{~V}$. Lower thresholds were used, since we observed a lower collection of photons per channel. In particular, the voltage discriminators $v$ th_t1, vth_t 2 and $v$ th_e were set to 4,8 and 8 DAQ units, respectively, meaning that the timestamp is generated at the first 1-3 photoelectrons. This set-up was also used during the calibration procedure designed to compensate the uncertainties in the timestamps introduced by the time-skew and time-walk errors, but also due to the SiPM energy non-linearity. The time-walk is referred to the dependency of the timing determination of a signal with its charge amplitude, while the time-skew refers to the timing error introduced by the different time-paths among ASIC channels, see Section 3.4 for further details.

Afterwards, two identical detectors, both based on monolithic blocks were tested in coincidence. It should be noted that for the experiments using the SiPM photosensors array, custom printed circuit boards (PCBs) were developed, as an interface between the DAQ boards and the SiPM arrays (see Fig. 2 top-right).

All measurements were carried out at stable temperatures environment $\left( \pm 0.5^{\circ} \mathrm{C}\right)$ in the range of 7 to $19^{\circ} \mathrm{C}$, depending on the experiment. Small temperature drifts may affect the results and, thus, special attention to this aspect was taken. Moreover, the whole assembly was placed inside a light tight box. $\mathrm{A}^{22} \mathrm{Na}$ source $(1 \mathrm{~mm}$ in diameter, $475 \mathrm{kBq}$ ) was used for all experiments. All results mentioned below, have been obtained after applying about 30\% (350-650 keV) energy window around the $511 \mathrm{keV}$ photopeak.
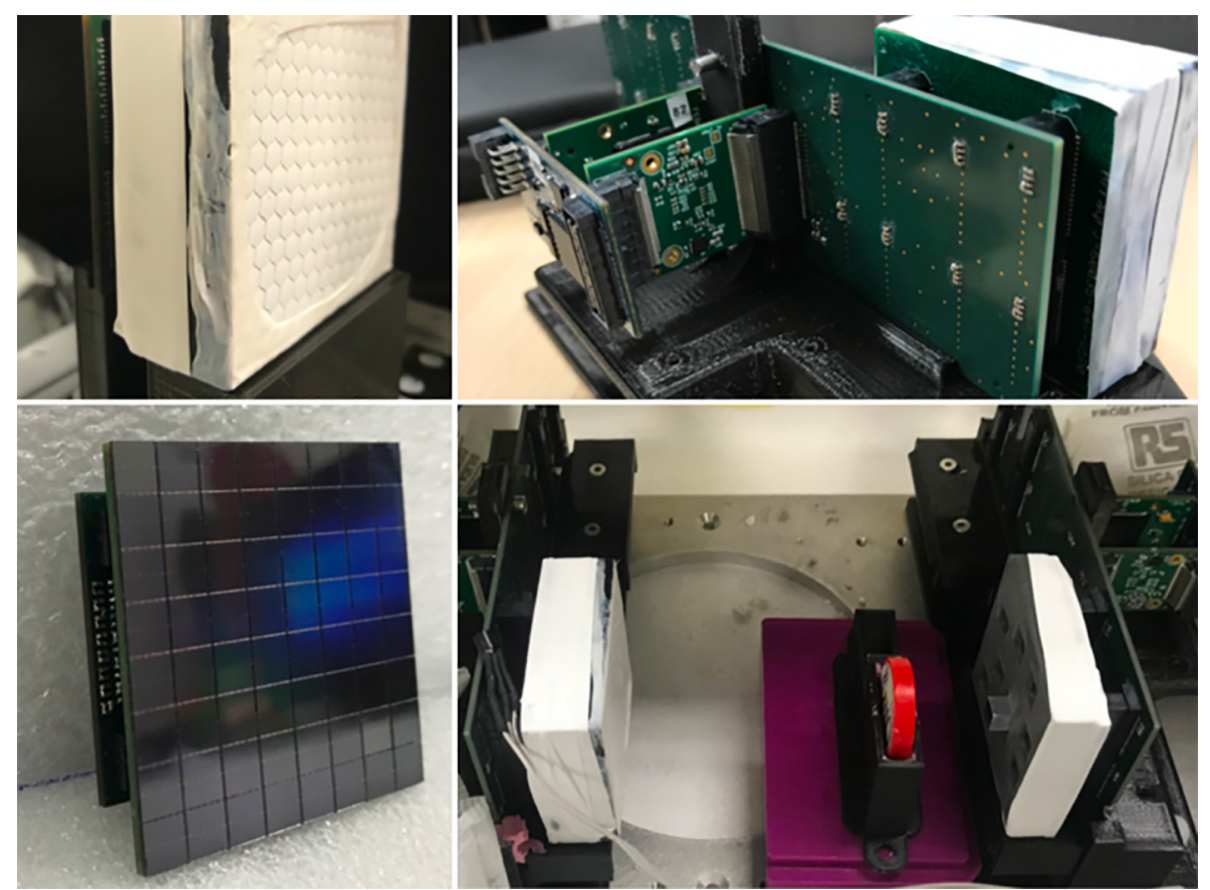

Fig. 2. Top-left, monolithic block $\left(50 \times 50 \times 15 \mathrm{~mm}^{3}\right)$ with a RR layer at the entrance. Top-right, TOFPET2 ASIC Front-End-Module. Bottom-left, SiPM $8 \times 8$ array with $6 \times 6 \mathrm{~mm}^{2}$ each photosensor element. Bottom-right, experimental set-up based on a monolithic block and a reference single pixel detector. 


\subsection{Analysis on the monolithic detector}

A simple Center-of-Gravity calculation was applied to estimate XY planar coordinates of each recorded gamma-ray event. Regarding the calculation of the $\mathrm{Z}$ coordinate, here referred as the Depth of Interaction (DOI), for each gamma-ray impact in the monolithic block we summed the energies collected for every row and column of the $8 \times 8$ SiPMs. Thereafter, the $\mathrm{Z}$ coordinate was determined using the estimator described as the ratio of the impact energy to the SiPM row (or column) to the highest signal [20]. The energy of each event is simply extracted by the sum of all channels fired.

For optimum timing determination, we have investigated an offline positioning filter. This means that an event is valid as long as the channels fired are in adjacent SiPMs (maximum of 8 therefore). In that way, false triggering due to SiPM dark counts can be rejected.

\subsection{Timing linearity tests}

The timing linearity of the system was studied with two detectors at a fixed distance, while the ${ }^{22} \mathrm{Na}$ source was moved across the field of view in between them. This experiment was carried out using the two monolithic blocks. We recorded the centroid of the timing distributions and compared the linearity observed from the measured experimental centroids and the expected values.

\section{Results}

\subsection{Pixelated crystals: one-to-one coupling}

The experiments with the KETEK PA3325 SiPMs and the small crystal pixels showed a CTR of 186 ps FWHM using default ASIC thresholds. Both detectors showed an energy resolution near $10.8 \%$ after correction for the SiPM saturation. Fig. 3 top shows both the energy plot and CTR histogram. The measurement was carried out at $19^{\circ} \mathrm{C}$. The tests were repeated using the $6 \times 6 \mathrm{~mm}^{2}$ photosensors and LYSO pixels of $6 \times 6 \times 15 \mathrm{~mm}^{3}$. Despite the larger active area of the photosensors that might introduce signal jitter due to larger capacitances, and the thickness of $15 \mathrm{~mm}$ of the LYSO pixels, a timing resolution of 330 ps FWHM was obtained (Fig. 3 bottom). For this set-up, the energy resolution was found to be $13.7 \%$ after again applying an energy calibration.
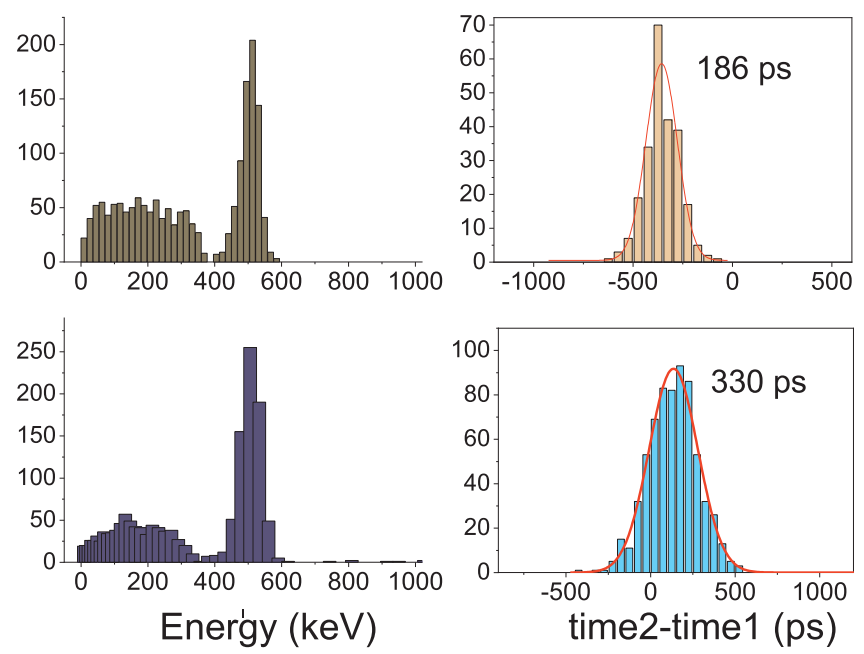

Fig. 3. Top, energy spectrum after energy calibration of one detector and time distribution obtained with $3 \mathrm{~mm}$ SiPMs and LYSO crystals of $3 \times 3 \times 5 \mathrm{~mm}^{3}$. Bottom, energy spectrum (after calibration) of one detector and time distribution obtained with $6 \mathrm{~mm}$ SiPMs and LYSO crystals of $6 \times 6 \times 15 \mathrm{~mm}^{3}$.
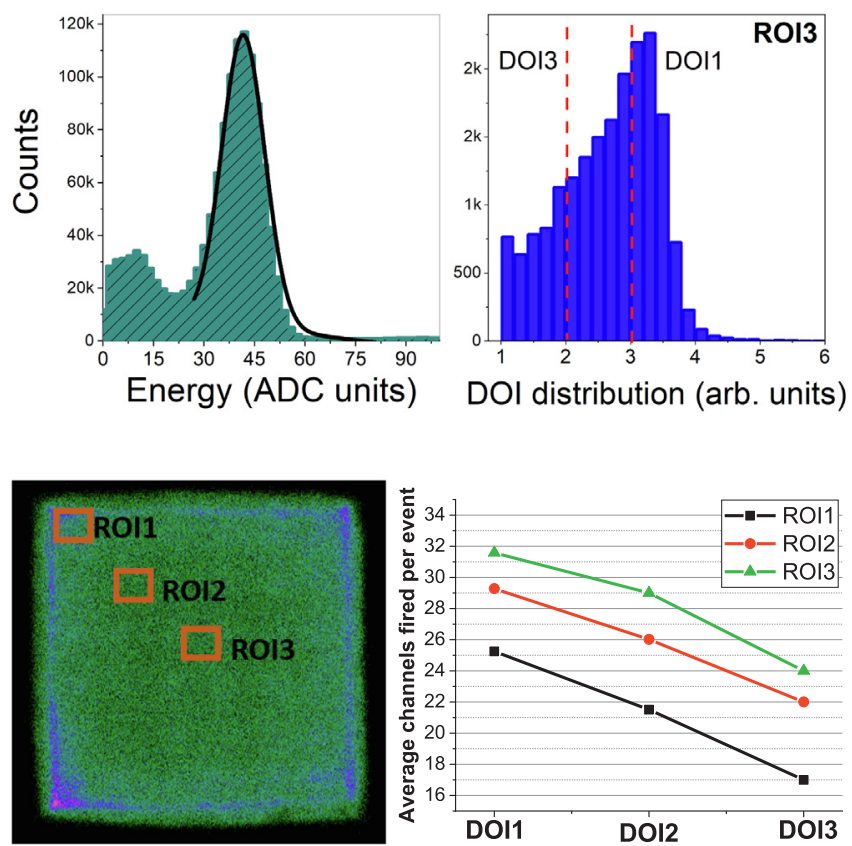

Fig. 4. Top-left, energy spectrum of the whole monolithic based detector before calibration. The black line shows a fit to the distribution using a Guassian profile plus a line. Top-right, DOI distribution of the events recorded at the center of the monolithic crystal (ROI3). Bottom-left, flood map of events, showing the three ROI selected for analysis. Bottom-right, average number of channels fired per event, as a function of the DOI and for the three ROIs.

\subsection{Monolithic blocks, light sharing}

The small size source was placed right in front of the reference detector and, therefore, the whole area of the monolithic crystal was irradiated during the coincidence measurements. An energy profile of all events in the monolithic crystal is shown in Fig. 4 top-left. Events within the photopeak (30-48 ADC units) were selected for data analysis. Three different Regions of Interests (ROIs) at the corner, middle and center of the detector block, were selected by applying a position filter, as depicted in Fig. 4 bottom-left. Moreover, for each ROI, the DOI distribution of events was obtained, allowing us to further split the data in three DOI regions (about $5 \mathrm{~mm}$ each) depending on the gamma ray impact Z coordinate. They are named as DOI1 for events at the crystal entrance, DOI2 for events occurring at the middle of the scintillator and DOI3 for events impinging at the bottom crystal layer (see Fig. 4 topright). Therefore, an estimation of the average number of channels that crossed the threshold and, hence, of the SNR per channel could be obtained for each gamma-ray impact. As seen in Fig. 4 bottom-right, we observed that independently of the XY position, a larger spread of the scintillation light was found for events at the upper crystal layers (DOI1). For impacts impinging deeper in the monolithic crystal e.g. DOI2 and DOI3, we can observe a slightly decreased number of channels fired, but still high suggesting a poor SNR per ASIC channel.

A significant dependency of the number of fired channels with the gamma-ray impact position is observed. The nearest an event occurred to the edge of the crystal, the more it suffers from light truncation as a high amount of scintillation photons are absorbed by the black painted walls. This fact explains the decreased number of channels fired for ROI1 and ROI2. It should be noticed, that these distributions are in general directly related to the dimensions and thickness of the crystal block as well as to the crystal treatment and photosensor geometry.

We have shown that the generated SNR per photosensor element strongly depends on the position of each particular event. Since an average of 25 channels are fired for each gamma-ray event, a poor SNR in the ASIC channels is expected. Gamma-ray impacts near the crystal 

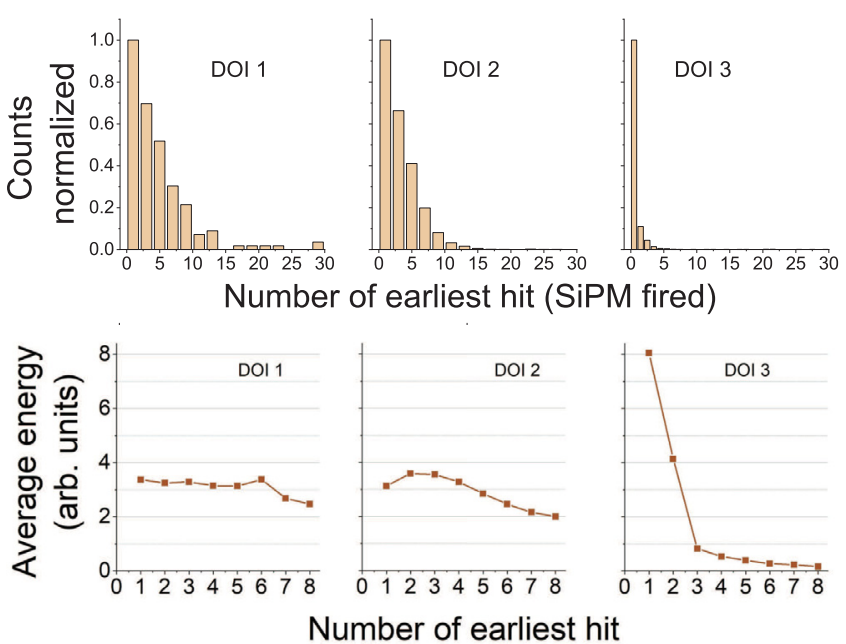

Fig. 5. Top, histograms showing which of hits collected the highest amount of energy for the three DOI regions for the whole scintillator. Bottom, average energy of each hit for all events recorded at the three DOI layers (no filter in position).

entrance (DOI1), which is the most probable scenario, will fire many photosensors but with a reduced number of collected scintillation photons per photosensor. This statement limits the basic TOF requirements, namely a short and sharp rise time of the signals [12]. On the contrary, events near the photosensor show a narrower light spread (DOI3), permitting a faster and a more efficient collection of optical photons. We shorted all impacts based on their timestamps and we used this information to fill the histograms shown in Fig. 5 top. Earliest hit 0 (X-axis of the histogram) means that the first timestamp also collected the maximum number of optical photons. Whereas for instance, hit labelled 10 means that the 10th impact collected the highest energy for this given gamma-ray event. Therefore, for gamma-ray impacts near the photosensor (DOI3), the channels collecting the highest amount of energy also correspond to the fastest ones (first hits). That is, we observe the hits with highest energy being the earliest collected. However, impacts at the crystal entrance exhibit a wider distribution of energy hits and time. This fact, was found to be directly related with the timing resolution.

Also interesting is the analysis of the energy ranges of the earliest channel triggered (earliest timestamp recorded) which complements the previously described behavior. By averaging the energies of the eight earliest hits for all events, it was clearly shown that the first recorded hit shows much higher energy ranges compared with the later recorded ones for the case of deep DOIs, while at the higher DOI1, the energy ranges for all 8 first hits are all comparable (Fig. 5 bottom). It should be noted, that these plots were obtained for the whole scintillator volume without using the previously described position filter. No significant variations are expected in these distributions for independent ROIs.

The variations in the spread of the scintillation light depending on the DOI of each gamma event lead to explore the optimal event timestamp assignment method [23]. When that many hits occurred for each event, it is critical to study if the optimal time resolution is given when using the first timestamp recorded of each event or an alternative approach is may needed.

\subsection{Monolithic detectors, time analysis}

When using the monolithic crystal and the reference pixel, the assembly was placed at a stable temperature environment of $7{ }^{\circ} \mathrm{C}$, minimizing dark count rates and increasing the Photon Detection Efficiency (PDE) of the photosensors. Coincidences measurements were carried

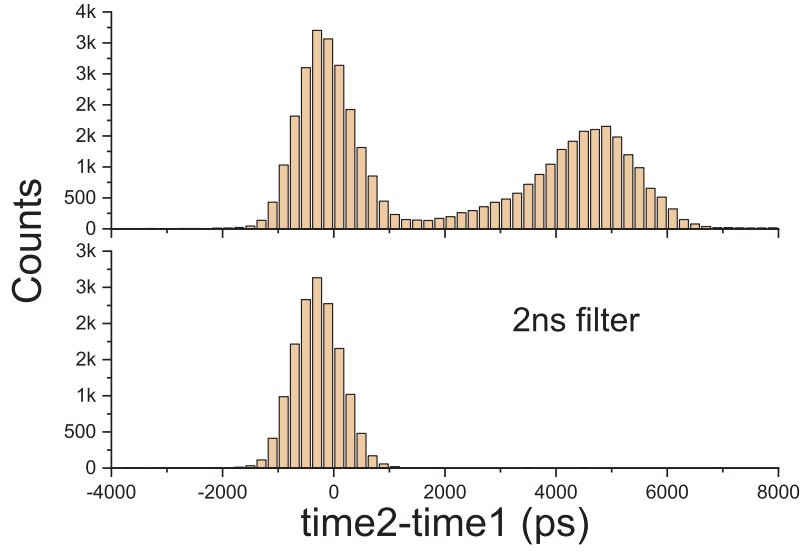

Fig. 6. Top, timing distribution of the measurement between the monolithic block and the reference detector without applying filtering windows. Bottom, timing distribution when applying a 2 ns window for the first six impacts.

out with the ${ }^{22} \mathrm{Na}$ source attached to the reference detector and data for the whole scintillator volume were obtained.

We first obtained the timing resolution using the timestamp of the channel with the highest energy, resulting on $1.41 \mathrm{~ns}$ FWHM. Alternatively, we sorted the data based on the timestamp and we used the earliest one recorded for the timing distribution. By plotting the difference of the timestamps, we observed an additional satellite peak centered at 5000 ps, see Fig. 6 top. The satellite peak is directly related to the overvoltage of the SiPMs as well as with the value of the vth $\mathrm{t} 1$ discriminator. Detailed analysis of this effect can be found in [28].

We applied a timing filter window accepting events whose first several hits recorded are within a time frame. In particular six hits were chosen as the optimum number of hits within this window. This filter had as a result an improvement of the CTR and the discard the satellite peak from the timing distribution plots, showing that this effect was a result of false triggering (Fig. 6 bottom). Table 1 summarizes the measured CTR for different filter timing windows. As it can be seen, narrower time windows significantly improve the CTR but also affect the statistics. Therefore, a window of $2 \mathrm{~ns}$ was selected and applied to all following measurements. This filter improved the measured time resolution to 996 ps FWHM.

Some authors have showed a significant CTR improvement when instead of the timestamp of the first hit, the timestamps of secondary hits are considered together with a low threshold at the level of the first photo-electron $[29,30]$. Fig. 7 shows experimentally the same behavior. When using the timestamp of the fourth recorded hit in time, the time resolution was improved. Herein, using this approach and the fourth arrived timestamp, we were able to reach to an improved CTR from 996 ps (RAW data) to 883 ps FWHM.

The timing resolution measured for this set-up is still influenced by the time-skew among the ASIC channels. Moreover, the time-walk also affects the CTR due to the poor collection of photons per photosensor element. Thus, a slower rising time is observed as a consequence of the scintillation light sharing effect.

Table 1

Table representing the CRT values as well as the statistics of the total event accepted for different filtering windows of the first 6 hits.

\begin{tabular}{ll}
\hline Filtering Window (ps) & CTR (FWHM, ps) \\
\hline 5000 & 1156 \\
4000 & 1133 \\
3000 & 1100 \\
2000 & 996 \\
1000 & 817 \\
\hline
\end{tabular}




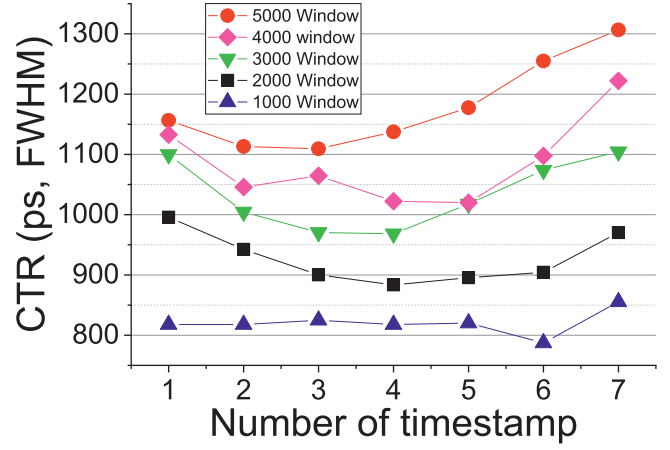

Fig. 7. Experimental results showing CRT measured as a function of the number of earliest timestamp used for different filtering windows.

\subsection{Time-skew and time-walk calibration}

The reference detector with the single LYSO pixel was placed at a distance of $25 \mathrm{~cm}$ from the monolithic detector and measured in coincidence mode. The ${ }^{22} \mathrm{Na}$ point source was attached to the reference detector aiming again to irradiate the whole volume of the crystal block and about $10^{6}$ events were recorded. Considering that the source, as well as the distance between detectors, remained constant during the experiment, the mean values of the timing distributions of all timestamp differences between all channels in the monolithic block and the reference one, should ideally be constant, independently of the energy collected.

Initially, aiming to obtain an estimation of the time-skew error, and not for calibration purposes, we selected events which occurred at the bottom of the crystal block and whose earliest recorded impacts contain a relatively high number of photons (8 ADC units). This filter was applied in order to consider only timestamps that are less influenced from noise. The aforementioned Gaussian mean values for the 64 pairs were obtained. These represent the time-skew for the 64 ASIC channels. Fig. 8 depicts the time offsets for all ASIC channels in this assembly. The introduced error can be as large as $1 \mathrm{~ns}$ when considering all channels for the CTR estimation.

In the following we describe the studies carried out regarding the time-walk influence. Fig. 9 left shows the timestamps differences for one single pair of channels as a function of the energy of the first hit recorded in the monolithic block before any calibration. Even when considering one single channel the timing resolution is strongly affected for lower energy impacts (see range 0 to 10 in arbitrary units), confirming the time-walk effect.

The 2D histograms containing the time differences as a function of the energy were generated for each channel of the monolithic detector

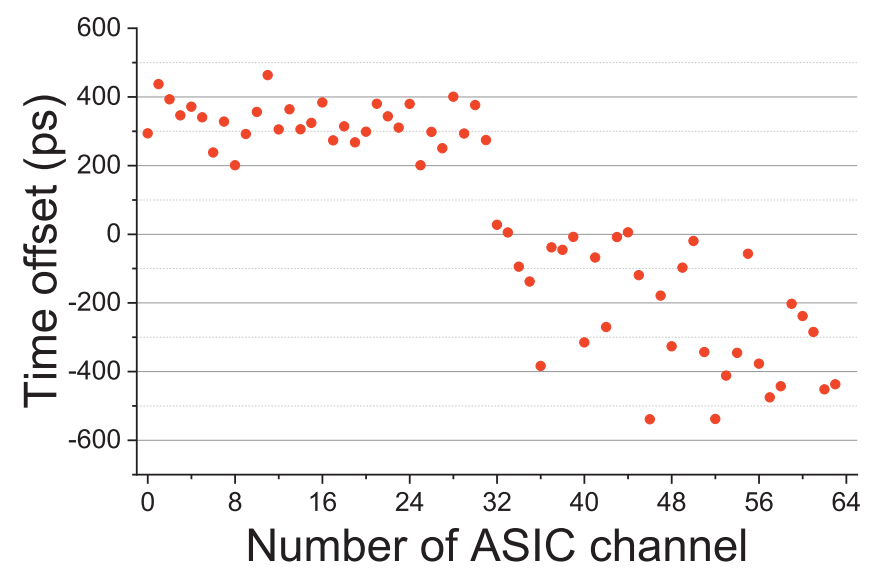

Fig. 8. Dispersion of Gaussian centroids of the time differences between the channels of the monolithic detector and the reference one (time-skew error).

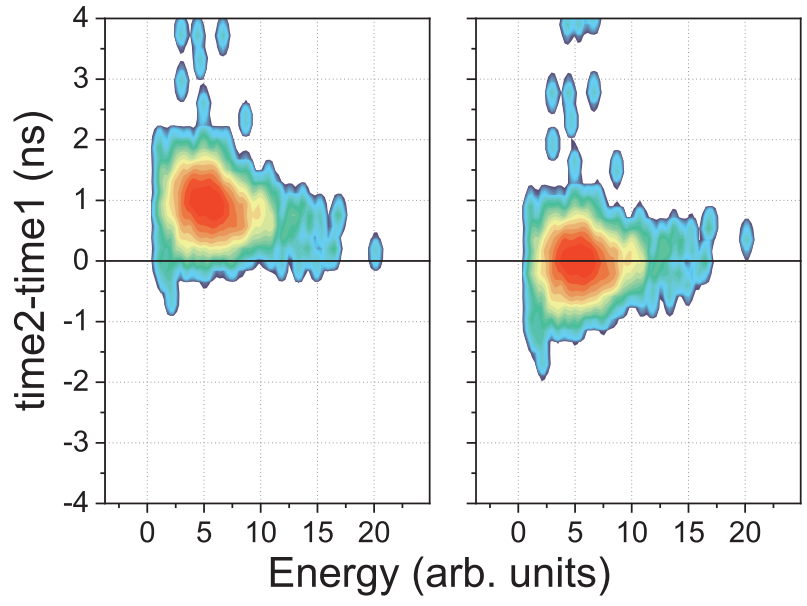

Fig. 9. Time differences of one channel as a function of the energy of the first hit before (left) and after the calibration (right). The color map is in logarithmic scale. (For interpretation of the references to color in this figure legend, the reader is referred to the web version of this article.)

(a total of 64). Then, they were fitted using a parabolic function and the fitting parameters stored in a Look-Up-Table. A parabolic function was used as it agrees well with the data behavior. The application of this method to all 64 channels of the monolithic based detector, besides some partial time-walk correction, also accounted for the time-skew errors, as all channel distributions were centered to zero (see Fig. 9 right). After correcting each recorded timestamp, an improvement of the CTR was observed for all channels, with the average value to be 851 ps FWHM, when using the earliest recorded timestamp.

The time arrival of secondary hits was again studied in detail, after applying all timestamp corrections. Fig. 10 shows the CTR when later recorded timestamps were used. A slighter improvement was observed when the second hit was used (black squares). However, we have also investigated the averaging of the timestamps $\left(\mathrm{t}_{\mathrm{i}}\right)$ of the few first hits and not just considering one. We have tested both a simple averaging of timestamps $\left(t_{\mathrm{SA}}\right)$ as well as an energy weighted average $\left(\mathrm{t}_{\mathrm{EA}}\right)$ :

$t_{S A}=\frac{\sum_{i=0}^{n} t_{i}}{n}, t_{E A} \frac{\sum_{i=0}^{n} t_{i} E_{i}}{\sum_{i=0}^{n} E_{i}}$

Up to eight timestamps were considered for both methods, see also Fig. 10, blue triangles $\left(t_{\mathrm{SA}}\right)$ and red circles $\left(\mathrm{t}_{\mathrm{EA}}\right)$, respectively. In particular, slightly optimum values were provided by the energy weighted average when the six earliest timestamps recorded were used, reaching about 580 ps FWHM. These values have been obtained for the whole

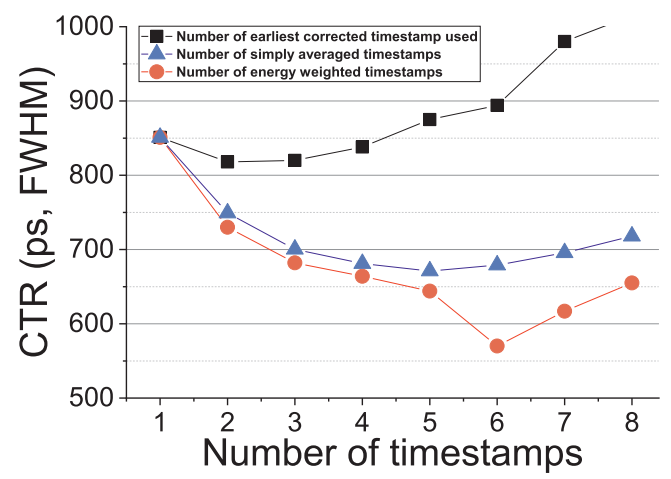

Fig. 10. Experimental CTR results (not position filter applied) using three methods; considering individually the eighth earliest timestam ps (black squares), a simple average of timestamps (blue triangles) and an energy weighted averaging (red circles). (For interpretation of the references to color in this figure legend, the reader is referred to the web version of this article.) 


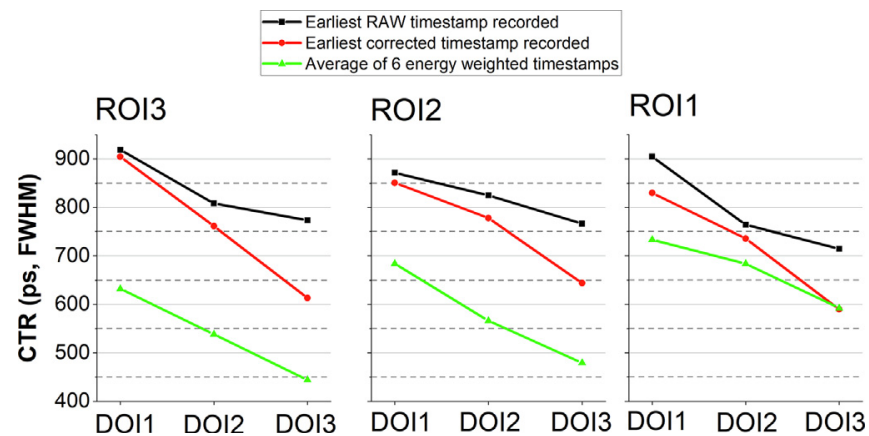

Fig. 11. Timing resolution CTR as a function of the DOI layer for the three ROIs, when using RAW timestamps, the earliest recorded timestamp corrected, and an energy weighted average of the earliest 6 corrected timestamps.

monolithic crystal volume.

Finally, after enabling the position filter mentioned in Section 2.4, an additional improvement in the CTR was found. The CTR improved from 580 ps to 550 ps FWHM. Since the contribution of the reference detector was estimated at $235 \mathrm{ps}$ FWHM $(330 / \sqrt{ } 2 \mathrm{ps})$, the resulting time resolution for the monolithic based detector was found to be $497 \mathrm{ps}$ FWHM.

\subsection{CTR dependency with $X Y$ and $Z$ position}

The three ROIs showed in Section 3.2 were selected for an independent and in deep detail analysis of the CTR performance based on the $X, Y$ and $Z$ position of the gamma-ray event. In all three ROIs when RAW timestamps are considered, the influence from the time walk and the poor SNR significantly affects the time resolution. This is especially observed for impacts at the crystal entrance layer (DOI1), as depicted in Fig. 11 with black squares. The same dependency, CTR vs DOI layer, is also found when only one corrected timestamp is used, but with some CTR improvement, as expected. However, when additional timestamps (six of them) are averaged using an energy weighting method the CTR is highly improved (green squares) and most importantly, its dependency with the DOI layer significantly decreases. Moreover, it is worth to highlight that in the case of events occurring near the crystal corner (ROI1) and at the bottom crystal layer (DOI3), the averaging method of timestamps seems to provide very similar results to the case of using just the earliest corrected timestamp. This behaviour can be expected from the fact that firstly, as already showed in Fig. 5, most of the collected scintillation photons occur in the first hit. Moreover, the scintillation light absorption by the black painted laterals limits the light spread. Besides this, no significant variations in the timing behaviour were found among ROIs, however best values were obtained for the case of the ROI3 and DOI3, resulting in a CTR value of 440 ps FWHM (371 ps FWHM when subtracting the contribution of the reference detector).

\subsection{Experiments with two monolithic blocks}

The two monolithic blocks were independently calibrated using the approach described above with a reference single-pixel detector. Then, they were measured in coincidence by placing the source in between the two detectors. Fig. 12 top shows the CTR values when considering an average of up to 8 timestamps (energy and simply average). Best timing resolution was achieved at 660 ps FWHM when using the sixth earliest timestamps weighted by energy. This data includes all impacts in the whole scintillation volume.

In order to validate the timing results, the linearity of the measured Gaussian centroids of the timing distributions was evaluated. In Fig. 12 bottom, the centroids obtained with the use of just one corrected timestamp as well as with the average of 6 timestamps weighted by the
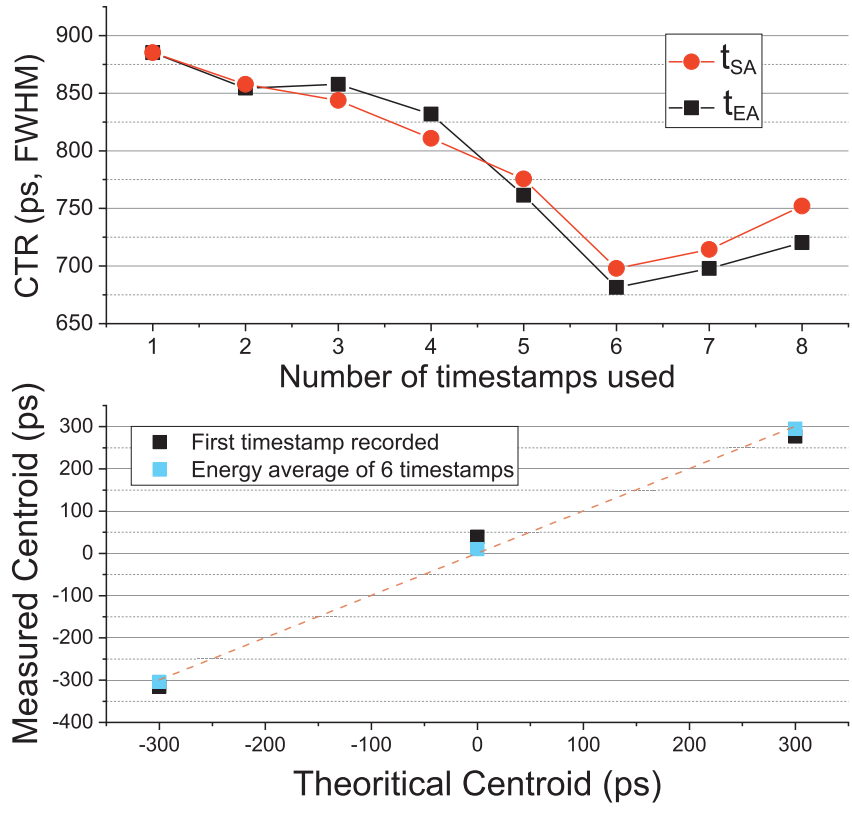

Fig. 12. Top, timing resolution of the coincidence measurement between the two monolithic detectors using simple timestamps average (red circles) and energy weighted timestamps average (black squares). Bottom, measured centroids as a function of the theoretical expected centroids using the earliest corrected timestamp (black squares) and an energy average of the 6 earliest timestamps (cyan squares). (For interpretation of the references to color in this figure legend, the reader is referred to the web version of this article.)

energy are plotted against the theoretical ones. Results shown that when using the first timestamp (black squares) a regression coefficient of 0.97 was obtained, while with the averaging method this was improved to 0.99 (cyan squares).

\section{Discussion}

The TOFPET2 ASIC is capable of resolving, with high accuracy, the gamma impacts in terms of timing and energy resolution. The experiments carried out with the small LYSO pixels ( $5 \mathrm{~mm}$ thick) exhibited state-of-the-art CTR values of $186 \mathrm{ps}$ FWHM using commercially available electronics. We hardly faced difficulties reaching this good timing, at even $19^{\circ} \mathrm{C}$ set-up temperature. However, when using thicker crystal pixels $(15 \mathrm{~mm})$ and larger photosensors of $6 \mathrm{~mm}$ size, a deterioration to $330 \mathrm{ps}$ was observed, as expected.

The use of a single pixel reference detector in the experiments with the monolithic crystals, permitted a better understanding of their timing performance. Here, the small crystal pixel minimizes the error introduced to the CTR determination.

An energy filter was applied to all measurements selecting events within the $511 \mathrm{keV}$ photopeak. Regarding the energy resolution in the monolithic based detector, it was found to be nearing $30 \%$ when considering all events within the whole crystal volume. However, this significantly improves when selecting small ROIs. For instance, for a ROI at the crystal center, an energy resolution of $17 \%$ was determined. The poor resolution obtained when considering all events independently of the position of the event, is caused by the effect of scintillation light truncation at the crystal edges. Moreover, the very low thresholds might produce some small deterioration in the energy resolution.

A parameter of significant importance on the timing resolution of the monolithic block was found to be the timestamp assignment. For comparison, in the case of pixelated crystals and the one-to-one coupling, the best CTR results are seen when using the timestamp of the hit with the highest energy, if more than one photosensor is fired (not 
shown in this work). However, this is no longer the case for monolithic based detectors. If the events occur near the photosensor, without producing any light sharing, the optimal approach would be to assign the timestamp of the first hit recorded, which is also the hit with the highest number of photons (energy) collected. However, in the case of events occurring in the entrance regions of the crystal, this approach cannot be applied, due to the low collection probability of enough photo-electrons in just one channel.

When there is an intense scintillation light sharing, very low thresholds are needed making the electronics sensitive to the very first photo-electrons. However, as we showed in this work, this low threshold may result in the acceptance of false triggered events, introducing uncertainty in the timing distribution. The timing window filter allowed us to discard noisy events and to improve the CTR. Although this filter significantly reduced the acquired statistics, we expect most of those rejected events are a result of false triggering. Moreover, by operating at very lower temperatures, a further reduction of the dark count rate will be achieved and, thus, discarding a smaller number of events. It should also be noted that the improvement seen by applying this filter is not related to the time-skew error as the improvement is also seen when only single pairs of channels are considered for the timing distribution, a method in which the time-skew has not effect.

It was observed that secondary hits provide better results in terms of timing. In some cases, an improvement better than 100 ps FWHM was observed. This behaviour has been studied in depth elsewhere and is directly related to the order and photocounting statistics [30]. As previous works have shown, when the optical photon index (i.e. hit number) increases, the time interval among the following detected photons is reduced. This means the probability distribution for detecting the first photon is significantly larger compared to the probability of determining the detection time of secondary photons. Therefore, it is hypothesized that the improvement seen in the experimental results is based on the timing generation probability of photons and in the order statistics theory, since there was no relation with the energy of each hit (as shown in Fig. 5 bottom).

A calibration procedure was carried out to correct each timestamp. We generated 2D plots of the time differences for each channel pair as a function of the impact energy. Here, we studied the time given using the timestamp of the first hit recorded, of the first 8 hits or of all hits. However, not significant differences were found among them, so the first hit was decided to be used in these plots. Instead of applying fits to the 2D plots (time difference vs. energy) for each channel, another method was formerly studied [26]. In that case, projections into the time difference axis were made in small energy steps. The centroids of the Gaussian-like profiles were used as timestamps offsets. However, since lower timing thresholds were currently used, the fitting approach described in this work showed slightly more accurate results.

Even after the calibration, the effect of the time-walk as well as of the false triggering might still be present. We expect some uncertainties in the generated timestamps, especially when the channel triggered did not collect a significant amount of scintillation photons. However, the method of averaging several timestamps, and in particular when weighting them by their collected energy, showed a significant improvement of the CTR, as it minimized the contribution of the noisy generated timestamps. This fact, was also verified when independently treating the CTR for different planar and DOI regions. As it was shown, the timestamp averaging method lead both to a partial compensation of the CTR and light spread dependency, providing more accurate CTR results than a single timestamp approach. The only difference was observed in the case of events occurring near the crystal edge and at the DOI layer near the photosensors. Here, we measured similar CTR both when using the averaging method or just a single timestamp. This might be explained due to the limited light spread in this region of the crystal. Herein, by analysing the CTR as a function of the DOI we were able to study the timing performance while avoiding the uncertainty introduced by the light speed propagation.

The CTR values obtained for the two monolithic blocks assembly were in accordance with the CTR recorded for the monolithic block in coincidence with the single pixel detector. We have estimated combined statistical and systematic error bars of about 20-30 ps FWHM. Notice that the custom PCB developed to interface the J-Series photosensors with the ASIC readout might introduce some additional noise to the signals coming from the photosensors due to their signals timepaths and higher capacitance. The validation of all methods used and described in the present manuscript was achieved with the linearity of the Gaussian centroids of three space-separated measurements.

\section{Conclusions}

We have evaluated the TOFPET2 ASIC showing its capability to achieve sub-200 ps FWHM time resolution using crystal pixels.

A thick and wide monolithic block was selected to be tested and explored in terms of timing resolution. The volume of the selected scintillation block exhibited several challenges in the determination of an accurate impact time resolution. The light sharing effect, and the resulting poor SNR per ASIC channel, is related to the size of the monolithic block. In addition to this, the selected treatment (black lateral paint and retroreflector layer at the entrance), on one hand enhances the determination of the impact coordinates, but on the other hand significantly degrades the timing resolution due to the scintillation light absorption at the lateral walls. We are aware these components somehow constrained the achieved performance, and that better absolute values could be obtained using smaller monolithic blocks, with white or reflecting painting, as well as when combined with photosensor arrays with smaller SiPM area. However, the analysis shown in this work is still useful to understand the overall limits and corrections to be applied when using monolithic blocks read out using analog SiPMs and ASICs. We have added especial focus in this work to the time-walk and the time-skew corrections.

The time-skew can be addressed through the independent processing of channel pairs but in the case of the monolithic block, the presence of time-walk uncertainties produces additional difficulties when aiming for an accurate calibration. Nonetheless, the calibration method described in this work provides good results. The time-skew was successfully corrected, permitting the exploitation of the timing information during future reconstruction processes. In addition, the time-walk has also been partially compensated, a fact that permits and motivates a follow up research work towards the development of TOF-PET detectors using other types and treatments of monolithic blocks.

Summarizing, RAW timing resolutions were found to be well above $1 \mathrm{~ns}$ for a large $50 \times 50 \times 15 \mathrm{~mm}^{3}$ LYSO block when tested in coincidence against a reference pixel-based detector. Techniques to discard a fraction of noisy events and decrease the time uncertainty were applied, reaching a significant improvement in terms of CTR of $550 \mathrm{ps}$ FWHM for the whole scintillation volume, without subtracting the reference detector contribution which is estimated at 230 ps FWHM. As shown in the analysis of the CTR and event position dependency, an improved timing resolution can be achieved for events at the center of the crystal and deep DOIs layers of 440 ps FWHM (again without subtracting the reference detector contribution). When two identical detectors were tested, CTR values of 660 ps FWHM were found. This timing resolution clearly cannot permit the use of timing information in the lines of response for small or organ dedicated systems [31], but will permit the reduction of noise as well as the improvement of the SNR in the reconstructed images. Moreover, recent pilot studies in our lab have shown to improve these results up to a factor of 2 if smaller crystals ( $1 \times 1$ in.), Teflon wrapped, and coupled to $8 \times 8$ SiPM arrays $\left(3 \times 3 \mathrm{~mm}^{2}\right)$ are used. 


\section{Acknowledgments}

This project has received funding from the European Research Council (ERC) under the European Union's Horizon 2020 research and innovation program (grant agreement No 695536). It has also been supported by the Spanish Ministerio de Economía, Industria y Competitividad under Grant TEC2016-79884-C2-1-R.

\section{References}

[1] Wong WH, Mullani NA, Philippe EA, Hartz RK, Gould KL. Imageimprovement and design optimization of the Time-of-Flight PET. J Nucl Med 1983;24:52-60.

[2] Gariod R, Allemand R, Carmoreche E, et al. The LETI Positrontomograph architecture and time of flight improvements. Proceeding of the Workshop on Time-ofFlight tomography. IEEE Publication: Washington University; 1982. p. 25-9.

[3] Wong Wong WH, Mullani NA, Philippe EA, Hartz RK, Bristow D, Yerian K, et al. Performance characteristics of the University of Texas TOFPET-I PET camera. J Nucl Med 1984;25:46-7.

[4] Surti S, Surti S. Update on time-of-flight PET imaging. J Nucl Med. 2015 Jan;56(1):98-105.

[5] Lecoq P. Pushing the limits in Time-Of-Flight PET imaging. IEEE Trans Rad Plasma Med Sci 2017:1. https://doi.org/10.1109/TRPMS.2017.2756674.

[6] Spanoudaki VC, Levin CS. Photo-detectors for time of flight positron emission tomography (ToF-PET). Sensors 2010;10:10484-505.

[7] Szczȩśniak T, Moszyński M, Świderski L, Nassalski A, Lavoute P, Kapusta M. Fast Photomultipliers for TOF PET. IEEE Trans Nucl Sci 2009;56:173-81.

[8] Renker D. 2007 New trends on photodetectors. Nucl Instr Meth Phys Res A 2007;571:1-6.

[9] Kim C, Wang GC, Dolinsky S. Multi-pixel photon counters for TOF PET detector and its challenges. IEEE Trans Nucl Sci 2009:56:2580-5.

[10] Frach T, Prescher G, Degenhardt C, Zwaans B. The digital silicon photomultiplier system architecture and performance evaluation. IEEE Nuclear Science Symposuim \& Medical Imaging Conference, Knoxville, TN. 2010. p. 1722-7.

[11] Frach T, Prescher G, Degenhardt C, De Gruyter R, Schmitz A, Ballizany R. The digital silicon photomultiplier - Principle of operation and intrinsic detector performance. 2009 IEEE Nuclear Science Symposium Conference Record (NSS/MIC), Orlando, FL. 2009. p. 1959-65.

[12] Moses WW. Current trends in scintillator detectors and materials. Nucl Instrum Meth A 2002;487(1-2):123-212.

[13] Gundacker S, Auffray E, Pauwels K, Lecoq P. Measurement of intrinsic rise times for various $\mathrm{L}(\mathrm{Y}) \mathrm{SO}$ and LuAG scintillators with a general study of prompt photons to achieve 10 ps. Phys Med Biol 2016;61(7):2802-37.

[14] Gundacker S, Acerbi F, Auffray E, Ferri A, Gola A, Nemallapudi MV, et al. State of the art timing in TOF-PET detectors with LuAG, GAGG and L(Y)SO scintillators of various sizes coupled to FBK-SiPMs. JINST 2016;11:P08008.
[15] Surti S, Karp JS. Advances in time-of-flight PET. Phys Med 2016;32(1):12-22.

[16] Gundacker S, Knapitsch A, Auffray E, Jarron P, Meyer T, Lecoq P. Time resolution deterioration with increasing crystal length in a TOF-PET system. Nucl Instr Methods 2014;737:9-100.

[17] Marcinkowski R, España S, Van Holen R, Vandenberghe S. Optimized light sharing for high-resolution TOF PET detector based on digital silicon photomultipliers. Phys Med Biol 2014;59(23):7125-39.

[18] González-Montoro A, Sánchez F, Martí R, Hernández L, Aguilar A, Barberá J, et al. Detector block performance based on a monolithic LYSO crystal using a novel signal multiplexing method. Nucl Instr Methods 2018;912:372-7.

[19] Xi D, Xie Q, Zhu J, Lin L, Niu M, Xiao P, et al. Optimization of the SiPM pixel size for a monolithic PET detector. Phys Procedia 2012;37:1497-2150.

[20] Gonzalez-Montoro A, Aguilar A, Canizares G, Conde P, Hernandez L, Vidal LF, et al. Performance Study of a Large Monolithic LYSO PET Detector With Accurate Photon DOI Using Retroreflector Layers. IEEE Trans Rad Plasma Med Sci. PP. 1-1. DOI: 10. 1109/TRPMS.2017.2692819.

[21] Krishnamoorthy S, Blankemeyer E, Mollet P, Surti S, Van Holen R, Karp JS Performance evaluation of the MOLECUBES $\beta$-CUBE - a high spatial resolution and high sensitivity small animal PET scanner utilizing monolithic LYSO scintillation detectors. Phys Med Biol 2018;63(15):155013.

[22] González-Montoro A, Sánchez F, Bruyndonckx P, Cañizares G, Benlloch JM, González AJ. Novel method to measure the intrinsic spatial resolution in PET detectors based on monolithic crystals. Nucl Instr Methods 2019;920:58-67.

[23] van Dam HT, Borghi G, Seifert S, Schaart D. Sub-200 ps CRT in monolithic scintillator PET detectors using digital SiPM arrays and maximum likelihood interaction time estimation. Phys Med Biol 2013;58(10):3243-57.

[24] Di Francesco A, Bugalho R, Oliveira L, Pacher L, Rivetti A, Rolo M, et al. TOFPET2: A high-performance ASIC for time and amplitude measurements of SiPM signals in time-of-flight applications. Journal of Instrumentation, vol. 11, no. 03, p. C03042.

[25] TOFPET2 ASIC Evaluation kit - Hardware User Guide (v1.2), v1.2, PETsys Electronics SA., 2018.

[26] Lamprou E, Aguilar A, González-Montoro A, Monzó JM, Cañizares G, Iranzo S, et al. PET detector block with accurate 4D capabilities. Nucl Instr Methods 2018;912:132-6.

[27] Acerbi F, Gundacker S. Understanding and simulating SiPMs. Nucl Instr Methods 2019;926:16-35.

[28] Schug D, Nadig V, Weissler B, Gebhardt P, Schulz V. Initial Measurements with the PETsys TOFPET2 ASIC Evaluation Kit and a Characterization of the ASIC TDC IEEE Trans Rad Plasma Med Sci. PP. 1-1. DOI: 10.1109/TRPMS.2018.2884564.

[29] Seifert S, Van Dam HT, Vinke R, Dendooven P, Löhner H, Beekman FJ, et al. A comprehensive model to predict the timing resolution of SiPM-based scintillation detectors: theory and experimental validation. IEEE Trans Nucl Sci 2012;59:190-204.

[30] Vinke R, Olcott PD, Cates JW, Levin CS. The lower timing resolution bound for scintillators with non-negligible optical photon transport time in time-of-flight PET. Phys. Med. Phys. Med. Biol. 59 6215. Phys Med Biol. 2014; 59(20): 6215-29.

[31] Gonzalez AJ, Sanchez F, Benlloch JM. 2018 Organ-Dedicated Molecular Imaging Systems. IEEE Trans Ratiat Plasma Med Sci. 2017; 2(5): 388-403. 